\title{
The parameterization of baroclinic instability in a simple model
}

\author{
by A. Wirth ${ }^{1}$
}

\begin{abstract}
Baroclinic instability of zonally forced flow in a two mode quasi-geostrophic numerical model with periodic boundary conditions is considered. Only the largest scale of the baroclinic mode is forced and the scale dependence of a diffusive parameterization of baroclinic layer thickness is determined. It is shown that the effect of baroclinic instability is a decreasing function of scale with an exponent of about half of that corresponding to the commonly used Laplace operator. We furthermore show that there is no linear relation between the time averaged amplitude of the large scale streamfunction (or quasi-geostrophicpotential vorticity) and the bolus velocity.
\end{abstract}

\section{Introduction}

One of the major challenges in ocean modeling is the parameterization of small-scale processes not explicitly resolved in the models themselves. Many attempts have been made in this direction. The difficulty arises from the nonlinear interaction of processes on a wide range of length and time scales.

Recently, interest has focused on the parameterization of a specific process, that is baroclinic instability. This process, of paramount importance in atmosphere and ocean dynamics, dominantly occurs at a small range of length and time scales. The typical length scale for the ocean is of a few times the baroclinic Rossby radius of deformation $(\approx 50 \mathrm{~km})$, while the typical time scale is of the order of tenth of days. This rather strong localization of baroclinic instability in wavenumber and frequency space might permit a successful parameterization.

More precisely, when using Global Circulation Models to determine the climate variability over several hundreds of years the grid resolution of the ocean models currently used is a few hundreds to several hundreds of kilometers. Such resolution is by far too poor to explicitly represent the effects of baroclinic instability on the large-scale motion and they have thus to be parameterized.

It is too ambitious to ask for a parameterization that exactly mimics the effects of the small scales on the larger ones. Two necessary conditions of such a parameterization would be: (i) that the dynamics of the small scales are completely slaved to the large scales, and

1. Institut fur Meereskunde, Universitat Kiel, Dusternbrooker Weg 20, D4105 Kiel, Germany. email: awirth@ifm.uni-kiel.de 
(ii) that the dimension of the chaotic attractor of the full problem is smaller than the number of degrees of freedom of our numerical model. Both conditions are unlikely to be satisfied. The parameterization should, however, approach the effects of the parameterized scales in a statistical sense.

A new way of parameterizing baroclinic instability was proposed by Gent and McWilliams (1990), based on diffusion of isopycnal thickness. Another, but not new, feature of the same parameterization is the down-gradient diffusion along isopycnals (see e.g. Redi, 1982). An interesting feature of this parameterization is that it can be interpreted as a quasi-adiabatic advection as explained in Gent $e$ t al. (1995). We like to refer the reader to this paper for a detailed discussion on the parameterization proposed by Gent and McWilliams.

There are different ways of analyzing such parameterization. A pragmatic approach is to implement such parameterization in a large-scale ocean model (Danabasoglu and McWilliams, 1995) and evaluate its performance. Another way is to verify the foundations of the theory in numerical experiments (Treguier, 1999).

The approach adapted here as in a variety of other experiments (see e.g. Killworth, 2000 and references therein) is to estimate the influence of the small scales on the larger ones in a fine resolution model, that is, a model resolving the Rossby radius of deformation. We like to emphasize here that for resolving a length scale $l_{0}$ it is not enough to have the grid-size $d_{0}$ of the order or slightly smaller than $l_{0}$. It is rather necessary that the length scale $l_{0}$ is in the inertial range of the nonlinear dynamics (see e.g. Frisch, 1996). This usually means that the grid size $d_{0}$ has to be chosen at least an order of magnitude smaller than the length scale $l_{0}$. It is indeed true that the dynamics on scales only a few times greater than the grid scale is dominated by linear dissipation being very different to the dynamics in the inertial range, which is dominated by nonlinear advection. The recent awareness of this problem in the ocean modeling community is apparent by the fact that models previously referred to as "eddy resolving" are now referred to as "eddy permitting."

The second point we like to dwell on is statistical significance. Our numerical results (see Section 4) show that even for the estimation of mean values and variances, that is the lowest order moments, averaging times of about a hundred years are necessary. When such long times are necessary for a parameterized quantity to relax to their mean value the results using such parameterization have to be handled with care. An immediate consequence is that parameterized models can only be interpreted in an ensemble sense.

The points mentioned in the previous two paragraphs put severe constraints on the feasibility of analyzing such parameterization. The experiment has thus to be set up very carefully, containing only the absolutely necessary ingredients. We thus consider the problem of "parameterizing baroclinic instability" in its numerically most feasible way. That is, we used a quasi-geostrophic two-mode model, which is periodic, both in the latitudinal and longitudinal direction. This is a simple model to test a parameterization of baroclinic instability.

Another important choice is the implementation of periodic boundary conditions. In 
previously published idealized experiments the dynamics far from the boundary appear to be completely slaved to the boundary conditions. Important quantities vary almost linearly between their extremal values at or near the boundary. Using periodic boundary conditions eliminates this problem completely.

A novelty of the present work is that a variety of experiments are performed with varying box-size, strength of forcing and viscous dissipation (model parameters) to estimate their (non-) influence on large-scale quantities like the diagnosed parameters for large-scale parameterization. It is indeed a crucial point of a large-scale parameterization that it should not, or only very weakly, depend on the above mentioned model parameters and this should be checked whenever a parameterization is proposed.

An advantage of testing parameterizations of baroclinic instability in a quasigeostrophic experiment is that in this simplified frame work the parameterization of layer thickness as proposed by Gent and McWilliams (1990) is identical to the parameterization of vertical mixing, as favored by Greatbatch and Lamb (1990). This means that the results presented here apply to the same extent to a whole class of parameterizations but also means that the results presented here can give us no hint to which parameterization in this class is better.

A disadvantage of the simplicity is that some of the important questions related to the parameterization of baroclinic instability cannot be addressed in this simplified frame work. One is the important effect on the long-term tracer dynamics (see e.g. Lee et al., 1997). The determination of the vertical dependence of a parameterization would require more baroclinic modes. We also neglect in our discussion the important point of the effect of baroclinic instability on the barotropic mode and the related question of how to implement vertical boundary conditions. For more details on this important point we refer the reader to Killworth (2000) and Treguier et al. (1997). The latter paper also contains a detailed discussion on eddy parameterization in quasi-geostrophic models. All the experiments presented here are restricted to the special case of purely zonal forcing. Thus, we could not consider the question of anisotropy of the diagnosed large-scale parameters as found by Rix and Willebrand (1996).

The next short section is devoted to the question of the compatibility between the $\beta$-plane approximation and periodic boundary conditions in both horizontal directions. In Section 3 we present the theoretical description of our numerical experiment which is introduced in Section 4. The results are then discussed in Section 5.

\section{The periodic $\beta$-plane}

This section may be skipped by people familiar with simulations on the doubly-periodic $\beta$-plane. We use spatially periodic boundary conditions in both horizontal directions. This is mathematically consistent with the $\beta$-plane approximation, for all evolution and diagnostic equations (see e.g. Hua et al., 1998, for a detailed discussion of this point).

A more subtle point, however, is the validity of the $\beta$-plane approximation in a domain having infinite extension in the meridional direction. The quasi-geostrophic potential 
vorticity is conserved along stream-lines of the quasi-geostrophically evolving geostrophic velocity field. When meridional boundaries are present at distance $L_{y}$, it is clear that the $\beta$-plane approximation is valid if $L_{y} \beta \ll f_{0}$. When no such boundaries are present, the condition for the $\beta$-plane approximation to be valid is that the distance a fluid parcel transported in the meridional direction, $L_{y}$, during a time-interval, $T_{0}$, by the geostrophic velocity field is small, $L_{y} \beta \ll f_{0}$. Where $T_{0}$ is the time length of the specific process under consideration, that is baroclinic instability in our case. This condition is usually verified as the $\beta$-term inhibits meridional-transport over large distances.

We are aware of the fact that the doubly periodic $\beta$-plane imposes some constraints on the dynamics. These constraints are somewhat of a different nature than those imposed by lateral walls, used in all other tests of baroclinic instability. Repeating the presented experiments in a channel geometry and comparing both would be very fruitful.

\section{The governing equations}

The equations governing the dynamics of this two-mode quasi-geostrophic model are,

$$
\begin{aligned}
\partial_{t} q_{1} & +J\left(\psi_{1}, q_{1}\right)+J\left(\psi_{2}, q_{2}\right)+\beta \partial_{x} \psi_{1} \\
& +b_{1} b_{1} \nabla^{2} \psi_{1}+b_{1} b_{2} \nabla^{2} \psi_{2}=-v \nabla^{6} \psi_{1}+w_{1} \\
\partial_{t} q_{2} & +J\left(\psi_{1}, q_{2}\right)+J\left(\psi_{2}, q_{1}\right)+\xi J\left(\psi_{2}, q_{2}\right)+\beta \partial_{x} \psi_{2} \\
& +b_{2} b_{1} \nabla^{2} \psi_{1}+b_{2} b_{2} \nabla^{2} \psi_{2}=-v \nabla^{6} \psi_{2}+w_{2},
\end{aligned}
$$

where $q_{i}$ represents the quasi-geostrophic potential vorticity of the $i$-th mode,

$$
q_{i}=\left(\nabla^{2}-\lambda_{i}^{2}\right) \psi_{i}
$$

$\psi_{i}$ is the corresponding streamfunction and $\lambda_{i}$ the inverse Rossby radius of deformation. The first mode, $i=1$, representing the depth-averaged velocity will be called barotropic and the second, $i=2$, baroclinic. The forcing is represented by the variables $w_{i}$, the bottom friction by $b_{i}$ and $\xi=1 / H \int_{-H}^{0} F_{2}(z)^{3} d z$ is the triple auto-interaction coefficient for the baroclinic mode, where $F_{2}(z)$ gives the vertical structure of the baroclinic mode. For further details the reader is referred to Flierl (1978) and Hua and Haidvogel (1986).

The (unphysical) parameter $v$ represents the dissipation at the smallest scales; its value is connected to the resolution of the numerical model and large-scale quantities such as the diagnosed parameters should be independent of $v$ when chosen in a sensible range.

We are now interested in the dynamics of the largest scale, $L$, of the model which is forced by $w_{2}=\overline{w_{2}} \sin \left(k_{0} y\right)$, where $k_{0}=2 \pi / L$. For that purpose we define the projection on this horizontal sine mode,

$$
\bar{A}=2 \int_{D} A \sin \left(k_{0} y\right) d x d y .
$$

It immediately follows from (3) that $\bar{\psi}_{i}=-\overline{q_{i}} /\left(k_{0}^{2}+\lambda_{i}^{2}\right)$. We choose $w_{1}=0$, as in most of the previously performed numerical experiments (Killworth 2000; Lee et al., 1997; 




Figure 1. The quantity $\overline{\partial_{y}\left\langle\left(\partial_{x} \psi_{1}\right) \psi_{2}\right\rangle} / \lambda_{2}^{-2} \overline{w_{2}}$ is plotted as a function of wavenumber for the six sets of experiments; experiments 1, 2, 3, 4, 5, 6 (see Table 1) as labeled, differences between the graphs are within statistical errors.

Treguier, 1999). This corresponds to thermal forcing as commonly used in atmospheric dynamics. Furthermore, we focus our attention on Eq. (2) as the perturbation of layer thickness is proportional to perturbations of $\psi_{2}$ when assuming a rigid lid at the surface. Applying (4) onto (2) we obtain,

$$
\begin{aligned}
-\left(k_{0}^{2}+\lambda_{2}^{2}\right) \partial_{t} \overline{\psi_{2}}-b_{2} b_{1} k_{0}^{2} \overline{\psi_{1}} & -b_{2} b_{2} k_{0}^{2} \overline{\psi_{2}} \\
& +\overline{J\left(\psi_{1}, q_{2}\right)}+\overline{J\left(\psi_{2}, q_{1}\right)}+\xi \overline{J\left(\psi_{2}, q_{2}\right)}=\nu k_{0}^{6} \overline{\psi_{2}}+\overline{w_{2}} .
\end{aligned}
$$

We suppose that the system is in a statistically stationary state, and that in the nonlinear terms the relative vorticity can be neglected which is a good approximation for scales larger than the baroclinic radius of deformation $\lambda_{2}^{-1}$. Averaging over time and keeping only the dominant terms we obtain:

$$
\left.\left\langle\overline{J\left(\psi_{1}, q_{2}\right)}\right\rangle \approx-\lambda_{2}^{2} \overline{J\left(\psi_{1}, \psi_{2}\right)}\right\rangle \approx \overline{w_{2}} .
$$

The subdominance of the two last nonlinear terms on the left-hand side of Eq. (5) and the above relation follows from simple scaling arguments when $k_{0} \rightarrow 0$, and is also verified numerically (see Section 4 and Fig. 1). The behavior of the baroclinic streamfunction at 
scales larger than the first baroclinic radius of deformation is to leading order identical to the behavior of a passive scalar advected by the barotropic velocity field and subject to a source $w_{2}$. This was already mentioned by Salmon (1980).

Using the mathematical identity,

$$
\left.\overline{\left\langle\left(\psi_{1}, \psi_{2}\right)\right.}\right\rangle=\overline{\partial_{y}\left\langle\left(\partial_{x} \psi_{1}\right) \psi_{2}\right\rangle},
$$

we then suppose that the following parameterization holds:

$$
-\lambda_{2}^{-2} \overline{w_{2}} \approx \overline{\left.\partial_{y}\left(\partial_{x} \psi_{1}\right) \psi_{2}\right\rangle}=\kappa^{(\alpha)}\left(-k_{0}^{2}\right)^{\alpha+1}\left\langle\overline{\psi_{2}}\right\rangle .
$$

When $\alpha=0$ the last equality represents the classical Gent-McWilliams parameterization as the perturbation of layer thickness is linearly related to the amplitude of the baroclinic mode in quasi-geostrophic theory.

In the above averaged equations the $\beta$-term has completely disappeared as the forcing and the averaged large-scale flow is zonal. The whole dynamics, however, depend on the $\beta$-term and so do the parameter values. An extreme example of this is to consider the case with $\beta=0$, where the dynamics are dominated by stable eddies that survive for very long times. This leads to a strongly intermittent behavior and no parameterization is reasonable in this case as time-averaged quantities relax too slowly to their mean value.

It is now easy to numerically measure the parameter $\alpha$ by determining the scale dependence of

$$
\kappa^{(\alpha)} k_{0}^{2 \alpha}=\frac{\overline{w_{2}}}{\lambda_{2}^{2} k_{0}^{2}\left\langle\overline{\Psi_{2}}\right\rangle} .
$$

The problem is thus reduced to determining the scaling law of the streamfunction average in the forced mode, $\sin \left(k_{0} y\right)$, as a function of the meridional wave number $k_{0}$,

$$
\left\langle\overline{\psi_{2}}\right\rangle \sim k_{0}^{-\gamma}
$$

where $\alpha=-1+\gamma / 2$.

\section{The numerical experiment}

When setting up the numerical experiment different constraints have to be considered:

(i) the results should be statistically significant,

(ii) the baroclinically most unstable modes should be in the inertial range,

(iii) the results should be compared for a variety of parameters.

The first constraint asks for long integration times, while the second requires high horizontal resolution. To satisfy all three points the experiment has to be carefully chosen. The results presented here are obtained by using Fourier series in the longitudinal and meridional direction. The nonlinear terms were treated using a pseudo spectral method (see 
Table 1. Overview of the parameters varied in the experiments performed.

Physical parameters

$\begin{array}{cc}\text { Exp. } & \bar{w}_{2} \\ 1 & 3.6 e-3 \\ 2 & 3.8 e-3 \\ 3 & 3.8 e-3 \\ 4 & 4.0 e-3 \\ 5 & 4.0 e-3 \\ 6 & 4.4 e-3\end{array}$

\section{Physical parameters}

$\lambda_{2} / k_{0}$
$9,9.5,10,10.5,11,12,13,14$
$10,11,13,14$
$9,9.5,10.5,11,12,13,14$
$9,9.5,10,10.5,11,12,13,14$
$9,9.5,10.5,11,12,13,14$
$8,9,9.5,10,10.5,11,12$

\begin{tabular}{lc}
\multicolumn{2}{c}{ Numerical parameters } \\
$v$ & Horizontal resolution \\
$1 . e-4$ & $128^{2}$ \\
$1 . e-4$ & $128^{2}$ \\
$1.2 e-4$ & $128^{2}$ \\
$1 . e-4$ & $128^{2}$ \\
$1.2 e-4$ & $128^{2}$ \\
$1 . e-4$ & $128^{2}$
\end{tabular}

e.g. Gottlieb and Orszag, 1977) and the resolution was 128 points in each horizontal direction.

In the vertical two modes, a barotropic and a first baroclinic, were used. The corresponding parameters for the nondimensionalized equations (1) and (2) are: $\lambda_{1}=2 \pi / \sqrt{1000}, \lambda_{2}=$ $2 \pi, \xi=15 / 4, b_{1}=4 . \cdot 10^{-2}, b_{2}=-1 \cdot \cdot 10^{-2}$ and $\beta=4.5 \cdot 10^{-2}$.

In dimensional parameters the baroclinic Rossby radius of deformation is $50 \mathrm{~km}$ while the average velocity in the forced large-scale baroclinic mode is about $6 \mathrm{~cm} / \mathrm{s}$. Velocities in eddies near the surface reach up to $100 \mathrm{~cm} / \mathrm{s}$. The value of $\beta$ corresponds to a latitude of about $39^{\circ}$. Each run covers at least 50,000 days of integration to insure statistical significance.

The largest scale $L$ which is also the forcing scale is chosen to be between 8 and 14 times the baroclinic Rossby radius of deformation. The "thermal" forcing is varied from $\overline{w_{2}}=$ $3.6 \cdot 10^{-3}$ to $4.4 \cdot 10^{-3}$. The hyper-viscosity parameter $v=1 . \cdot 10^{-4}$ and $1.2 \cdot 10^{-4}$ was varied to check that the results are independent from this unphysical parameter (being a function of the numerical resolution). An overview of the model parameters varied in the numerical experiments can be found in Table 1.

In Figure 1 it can be clearly seen that the approximation in the left part of Eq. (7) is very well verified for the scales chosen here, and that it deteriorates with decreasing scale separation, when the forcing scale becomes comparable to the Rossby radius of deformation. In Figure 2 the scaling behavior of $\left\langle\overline{\psi_{2}}\right.$ versus the forcing scale can be seen showing that for scales of about 10-times the baroclinic Rossby radius of deformation the scaling is close to $k^{-1}$ and clearly not equal to $k^{-2}$. This can be seen more clearly in Figure 3 where the parameter $\kappa^{(0)}$ is plotted versus wavenumber suggesting that $\gamma \approx 13 / 12$. This leads to a value of $\alpha \approx-11 / 24$. This value is only a good fit to the numerical data but it shows that we clearly do not have normal diffusion $(\gamma=2)$ and also that the transport is close to, but different from, ballistic transport $(\gamma=1)$.

In the different sets of numerical experiments we also varied the forcing by $\pm 10 \%$ and found about the same variation in $\kappa$ (see Fig. 1), while $\left\langle\overline{\psi_{2}}\right\rangle$ showed no variation (see Fig. 2). The reason is that for increased forcing (see Fig. 1) baroclinic instability occurs more often (Fig. 4), leading to a linear increase in the bolus velocity while the average streamfunction amplitude of the forced mode $\overline{\psi_{2}}$ stays almost constant. This behavior can be verified in 


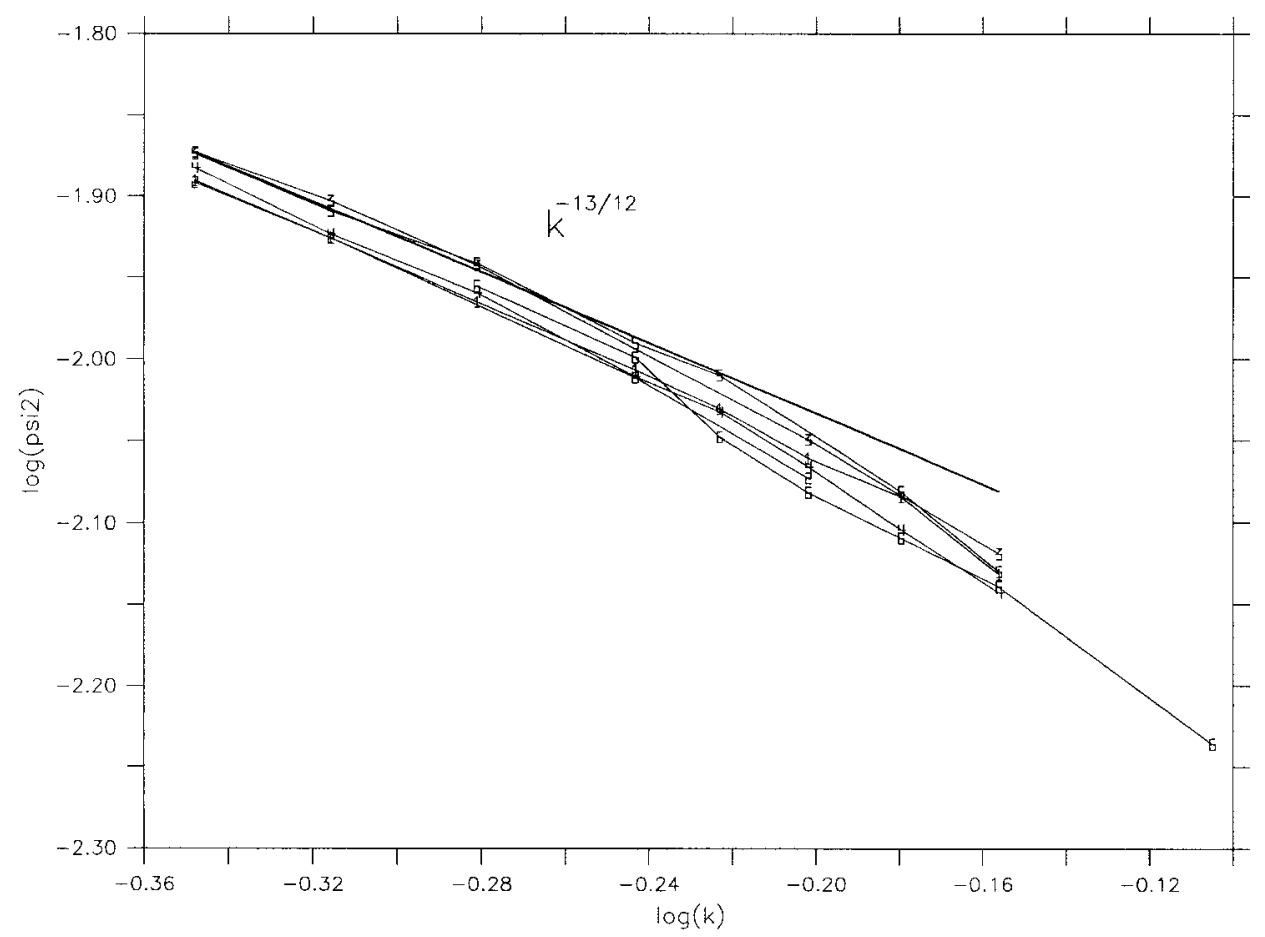

Figure 2. Scale dependence of $\overline{\left\langle\psi_{2}\right\rangle}$ for the six sets of experiments as labeled, differences between the graphs are within statistical errors.

Figure 4 where the strongly smoothed temporal energy spectrum of $\overline{\psi_{2}(t)}$ is plotted for three experiments having the largest scale that is 11-times the baroclinic Rossby radius of deformation and differing only in the forcing amplitude $\overline{w_{2}}$. It can be clearly seen that although the mean value $\left\langle\bar{\psi}_{2}\right\rangle$ is unchanged by the forcing (Fig. 2), the temporal energy spectrum is larger and peaked at higher frequencies when forcing increases. These findings are not astonishing and explained by the fact that baroclinic instability occurs at a critical shear. When applying a stronger forcing this critical shear is reached in a shorter time. Similar conclusions are obtained in other publications on slightly different subjects (see e.g. Straub, 1993).

We also increased the (nonphysical) lateral friction coefficient $v$ by $20 \%$ and found a decrease of $\kappa^{0}$ by about $3 \%$ (see Fig. 3). This slight dependence should disappear when using even higher spatial resolutions and lower lateral friction coefficients $v$.

\section{Discussion}

The above results force us to rethink some concepts of parameterizing baroclinic instability, as they demonstrate that: (i) the effect of baroclinic instability on larger and larger scales decreases slower than predicted by a Laplace operator and furthermore, (ii) 


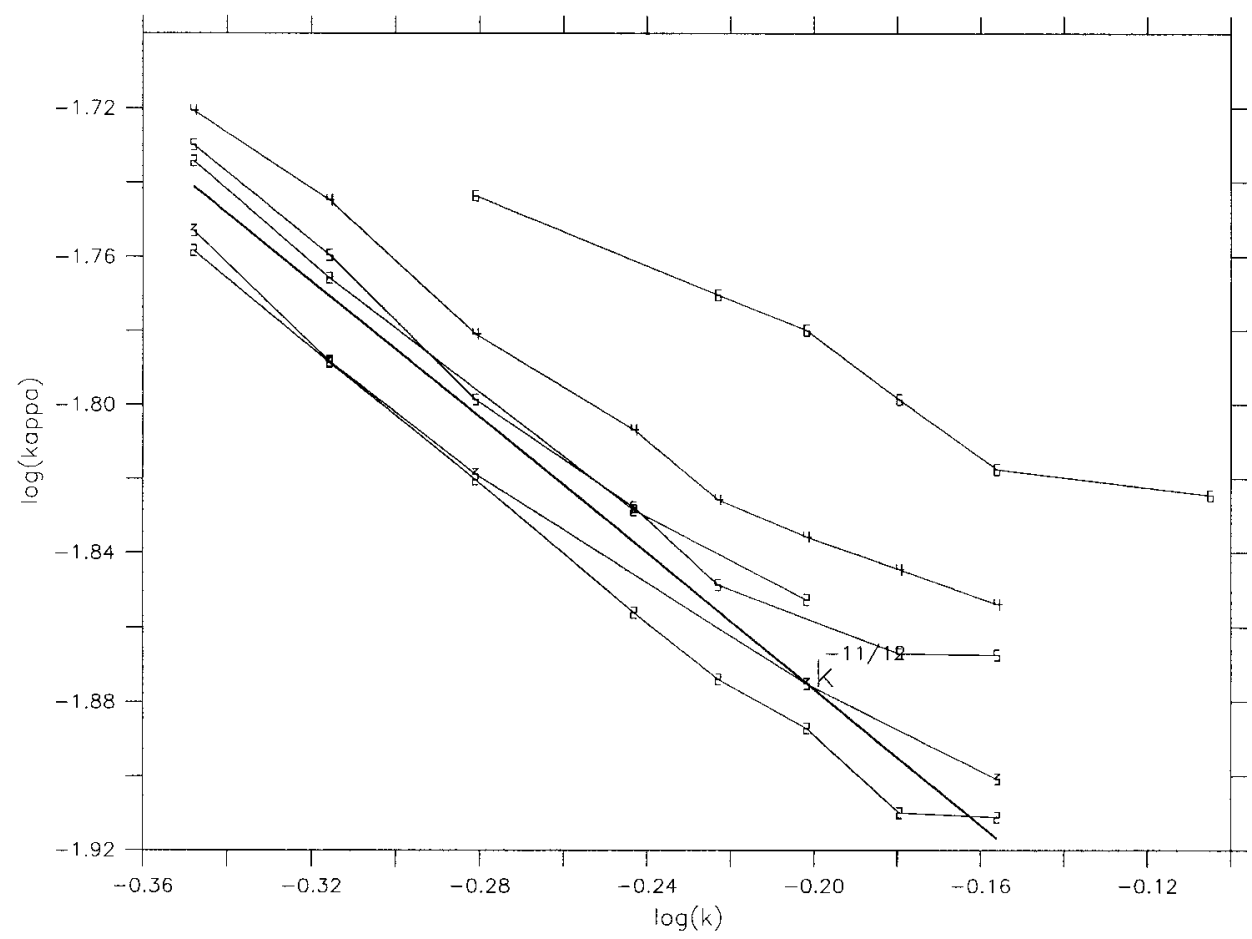

Figure 3. Scale dependence of $\kappa^{(0)}$ (obtained using Eq. (9)) for the experiments 6, 4, 5, 2, 3, 1 from top to bottom.

the bolus velocity grows linearly with the amplitude of the forcing, while the amplitude of the baroclinic streamfunction does not vary.

The latter findings seem to contradict the results of Rix and Willebrand (1996) who found a reasonable fit for linear relation of "layer-thickness" versus bolus velocity in a primitive equation North Atlantic model, when averaging results over $4^{\circ} \times 4^{\circ}$ boxes. However this is entirely due to the fact that we show results as a function of the ratio scale/(baroclinic Rossby radius of deformation). In the calculations by Rix and Willebrand (1996) a primitive equation model is used having many levels and a variety of baroclinic Rossby radii, unlike our simple model possessing only one. They also performed averages over different areas and seasons having different baroclinic Rossby radii of deformation. We produced a similar plot with our data. In Figure 5 we show the bolus velocity plotted against the amplitude of the forced mode weighted by the appropriate power of the streamfunction, that is: $\overline{\psi_{2}} / k^{1 / 12}$. To summarize this point we can say that although there is no linear relation between the bolus velocity and the baroclinic large-scale streamfunction when all other model parameters are kept constant, this relation can be found in a statistical sense when averaging over data from regions with different and a variety of baroclinic Rossby radii of deformation. 


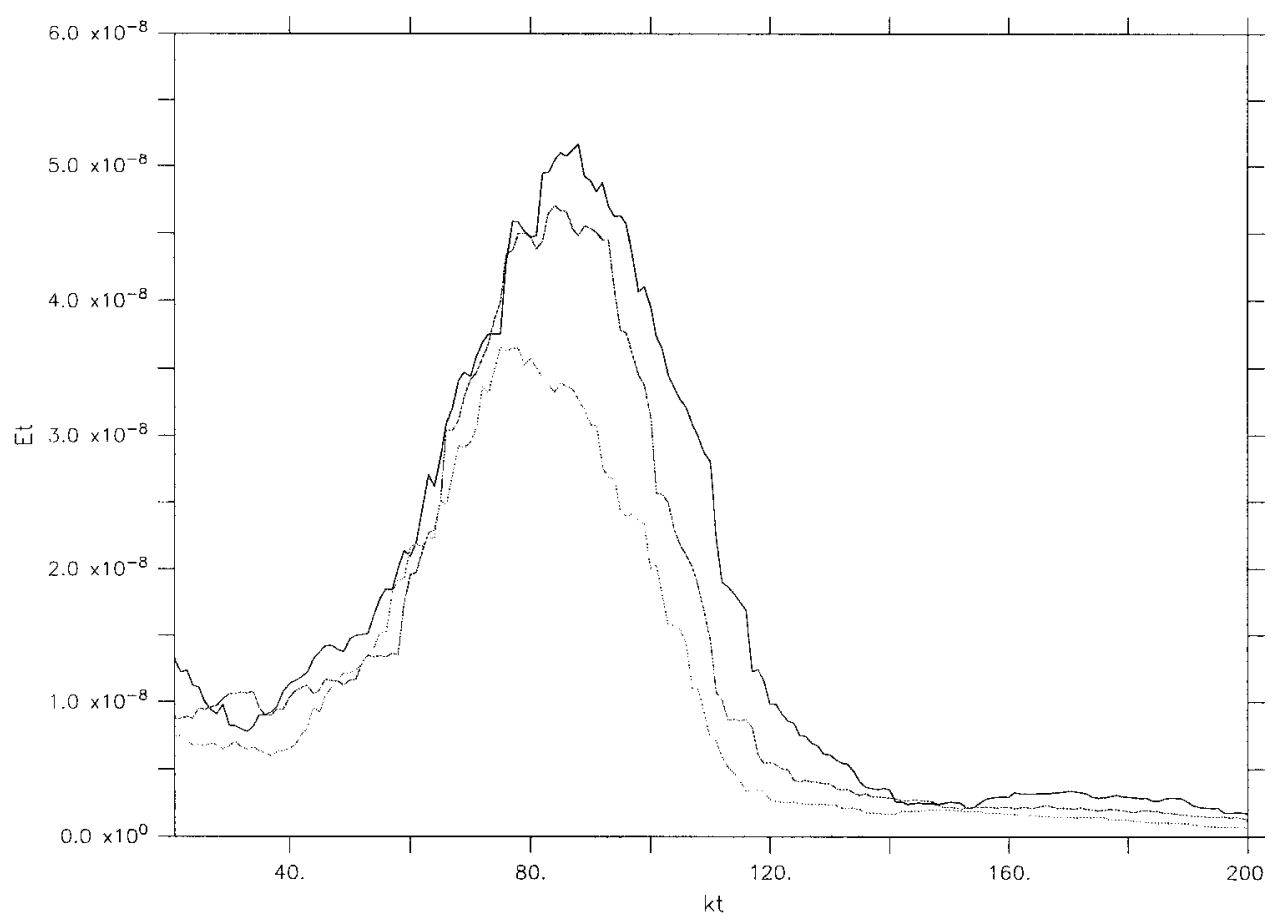

Figure 4. Temporal energy spectrum of $\overline{\psi_{2}}$ for $k=2 \pi \cdot 11$ in three experiments 1,2 and 4 (from top to bottom) after a 35-point-wide boxcar smother was applied. The peaks correspond to the time scale of about 200 days.

The first point, (i), saying that the effect of baroclinic instability on the baroclinic large-scale gradient is super diffusive seems to contradict intuition. The intuitive picture is indeed that the dynamics at the (small) scale of the baroclinically most unstable mode has a diffusive effect on the large-scale gradient. The point, however, is that energy injected in the barotropic mode does not stay at such small scales but cascades to the large scales, as explained by the two-dimensional inverse energy cascade (Kraichnan, 1967). The effect of this barotropic large-scale dynamics, caused by baroclinic instability, on the baroclinic large-scale gradient has to be parameterized as a super diffusive behavior. It is indeed well known that a lack of scale separation between the "large" scale and the parameterized scales lead to super diffusive behavior (Avellaneda and Majda, 1992). The inverse cascade is, however, halted by the $\beta$-effect at the Rhines scale; that is, the scale at which the meridional change of the Coriolis parameter balances nonlinearity (see e.g., Rhines, 1975, and Held and Larichev, 1996). This indicates that a normal diffusive parameterization might be adapted for scales much larger than the Rhines scale, that is for scales on the order of thousands of kilometers. Calculations of much higher resolution would be needed to determine such behavior. This also shows that for the practical use of parameterizing baroclinic instability in non-eddy-permitting ocean models and climate models, a super 


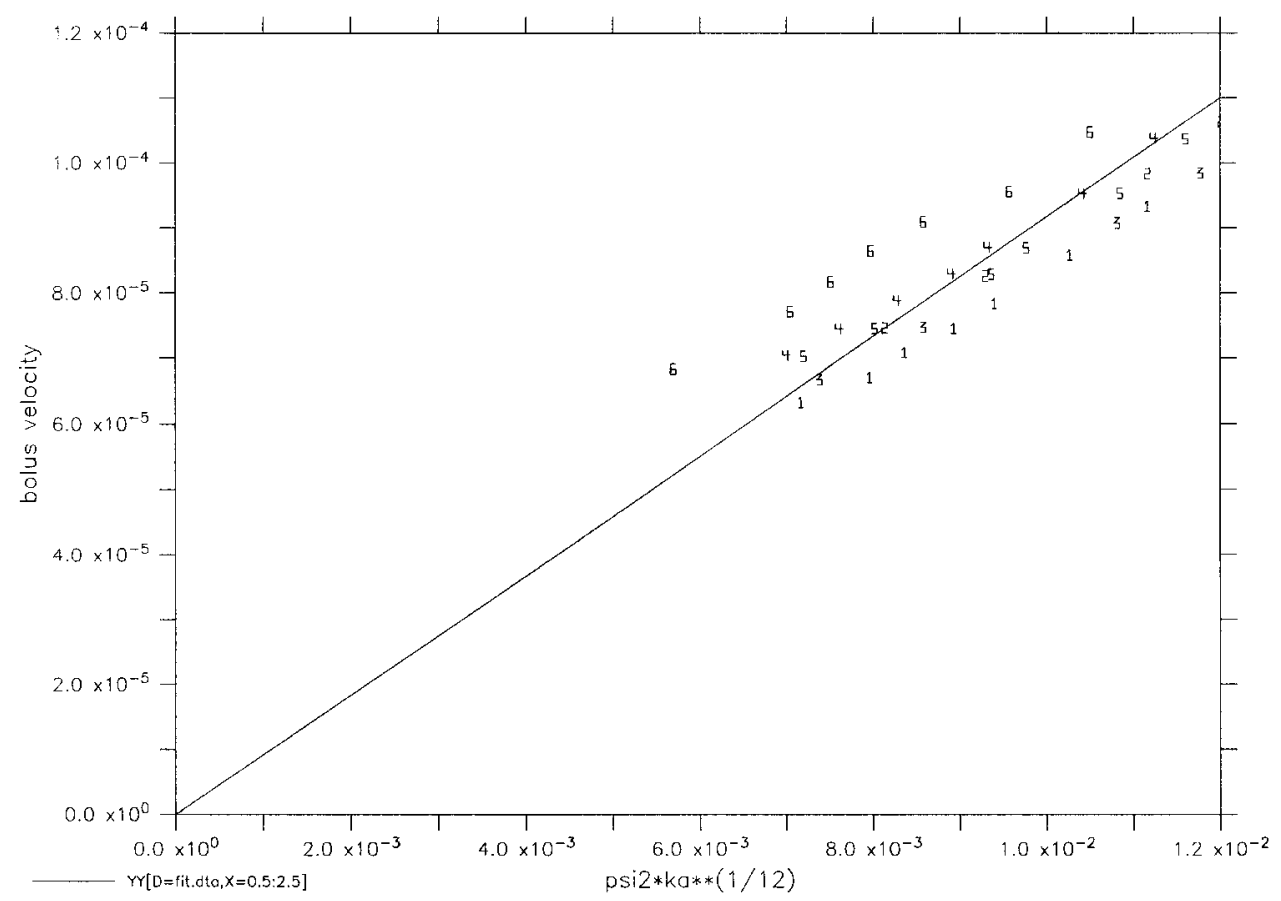

Figure 5. Bolus velocity versus $\left\langle\bar{\psi}_{2}\right\rangle k^{1 / 12}$.

diffusive parameterization should be more adaptable as their small scales range from roughly $100 \mathrm{~km}$ to $1000 \mathrm{~km}$. These are the scales where parameterization acts strongest and these are the scales considered in this paper.

The lack of scale separation is only one possible source for super diffusive behavior; spatio-temporal correlations between the barotropic and baroclinic dynamics might be another one.

For the case of the $f$-plane it was recently shown by Gryanik et al. (2000), that vortex-dominated transport is indeed ballistic. The situation on the $\beta$-plane considered here is somehow different as no such coherent structures exist.

To conclude we have to address the question of how good do the previously used parameterizations do and what is a better parameterization in view of the above presented results? In non-eddy-resolving ocean models one should include some kind of sink for layer thickness or baroclinic potential vorticity and most parameterizations can be seen as a first-order approach. Using a linear relation between the large-scale layer thickness and the bolus velocity is a simple and reasonable approach at least in a statistical sense. A more serious point is the commonly used diffusive law for layer thickness or potential vorticity which is here shown to be wrong. Implementing a dissipation scheme that represents a fractional power of the Laplacian is, however, cumbersome in the framework of finite differences. Please note that this is different from using a coefficient that depends on the 
local deformation rate as introduced into ocean modeling by Smagorinsky (1963). The former represents a linear operation, while the latter is not.

We emphasize once more that the experiments presented here are done with a simple model and generalizations to ocean general circulation models should be taken with care. In this sense this paper does not propose a new parameterization based on the above findings nor does it indicate which previously introduced parameterization does best. Rather, the purpose of this paper is to point out major difficulties in the parameterization of baroclinic instability that were not mentioned in previous discussions.

Acknowledgments. I am grateful to J. C. McWilliams and J. Willebrand for extensive discussions and to an anonymous referee for remarks that helped to improve the paper.

\section{REFERENCES}

Avelaneda, M. and A. J. Majda. 1992. Super diffusion in nearly stratified flows. J. Stat. Phys., 69, 689-729.

Danabasoglu, G. and J. C. McWilliams. 1995. Sensitivity of the global ocean circulation to parameterizations of mesoscale transports. J. Climate, 8, 2967-2987.

Flierl, G. R. 1978. Models of vertical structure and the calibration of two-layer models. Dyn. Atmos. Oceans, 2, 341-381.

Frisch, U. 1996. Turbulence: The Legacy of A. N. Kolmogorov, Cambridge University Press, 296 pp.

Gent, P. R. and J. C. McWilliams. 1990. Isopycnal mixing in ocean circulation models. J. Phys. Oceanogr., 20, 150-155.

Gent, P. R., J. Willebrand, T. J. McDougall and J. C. McWilliams. 1995. Parameterizing eddyinduced transports in ocean circulation models. J. Phys. Oceanogr., 25, 463-474.

Gottlieb, D. and S. Orszag. 1977. Numerical analysis of spectral methods: theory and applications, SIAM, Philadelphia, PA.

Greatbatch, R. J. and K. G. Lamb. 1990. On parameterizing vertical mixing of momentum in non-eddy-resolving ocean models. J. Phys. Oceanogr., 20, 1634-1637.

Gryanik, V., T. Doronina, D. Olbers and T. Warnecke. 2000. The theory of 3D hetons and vortex dominated spreading in localized convectionin a rotating stratified fluid. J. Fluid Mech., (in press).

Held, I. M. and V. D. Larichev. 1996. A scaling theory for horizontally homogeneous, baroclinically unstable flow on a beta plane. J. Atmos. Sci., 53, 946-952.

Hua, B. L. and D. B. Haidvogel. 1986. Numerical simulation of the vertical structure of quasigeostrophic turbulence. J. Atmos. Sci., 43, 2923-2936.

Hua, B. L., J. C. McWilliams and P. Klein. 1998. Lagrangian accelerations in geostrophicturbulence. J. Fluid Mech., 366, 87-108.

Killworth, P. D. 2000. Boundary conditions on TRM velocities in parameterisations. J. Phys. Oceanogr. (in press).

Kraichnan, R. H. 1967. Inertial ranges in two dimensional turbulence. Phys. Fluids, 10, 1417-1423.

Lee, M., D. P. Marshall and R. G. Williams. 1997. On the eddy transfer of tracers: advective or diffusive? J. Mar. Res., 55, 483-505.

Redi, M. H. 1982. Oceanic isopycnal mixing by coordinate rotation. J. Phys. Oceanogr., 12, $1154-1158$.

Rhines, P. 1975. Waves and turbulence on a beta-plane. J. Fluid Mech., 69, 417-433.

Rix, N. H. and J. Willebrand. 1996. Parameterization of mesoscale eddies as inferred from a high-resolution circulation model. J. Phys. Oceanogr., 26, 2281-2285. 
Roberts, M. and D. Marshall. 1998. Do we require adiabatic dissipation schemes in eddy-resolving ocean models? J. Phys. Oceanogr., 28, 2050-2063.

Salmon, R. 1980. Baroclinic instability and geostrophic turbulence. Geophys. Astrophys. Fluid Dyn., $15,11-37$.

Smagorinsky, J. 1963. General circulation experiments with the primitive equations: I. The basic experiment. Mon. Wea. Rev., 91, 99-164.

Straub, D. N. 1993. On the transport and angular momentum balance of channel models of the Antarctic Circumpolar Current. J. Phys. Oceanogr., 23, 776-782.

Treguier, A. M. 1999. Evaluating eddy mixing coefficients from eddy-resolvingocean models: A case study. J. Mar. Res., 57, 89-108.

Treguier, A. M., I. M. Held and V. D. Larichev. 1997. Parameterization of quasi-geostrophiceddies in primitive equation ocean models. J. Phys. Oceanogr., 27, 567-580.

Welander, P. 1973. Lateral friction in the ocean as an effect of potential vorticity mixing. Geophys. Fluid Dyn., 5, 173-189. 